\title{
Inhibition of the activating signals in NK92 cells by recombinant GST-sHLA- G1 $\alpha$ chain
}

\author{
Ai Yu YAO ${ }^{1,2}$, Hai Yang TANG ${ }^{1}$, Yun WANG ${ }^{1}$, Mei Fu FENG ${ }^{1, *}$, Rou Li ZHOU ${ }^{2, *}$ \\ ${ }^{1}$ State Key Laboratory of Biomembrane and Membrane Biotechnology, Institute of Zoology, Chinese Academy of Science, \\ Beijing 100080, China \\ ${ }^{2}$ Department of Cell Biology and Medical Genetics, School of Basic Medical Sciences, Health Science Center, Peking \\ University, Beijing 100083, China.
}

\begin{abstract}
The soluble HLA-G1 (sHLA-G1) isoform was found to be secreted by trophoblast cells at the materno-fetal interface, which suggests that it may act as an immunomodulator during pregnancy. In this paper, we reported that GST-sHLAG1 $\alpha$ chain could bind to its receptor ILT-2 on NK92 cells and then the latter recruited Src homology 2 domaincontaining tyrosine phosphatase-1 (SHP-1), which consequently dephosphorylated some important protein tyrosine kinases and blocked the activation of downstream molecules such as MEK and ERK so that the cytotoxicity of natural killer (NK) cells was inhibited. These results indicated that GST-sHLA-G1 $\alpha$ chain might be exploited in new immunotherapy strategies aiming at inducing immunotolerance during allograft, xenograft and autoimmune situations. In addition, we found that modification of O-linked $\beta$-N-acetylglucosamine (O-GlcNAc) was involved in NK cells' activating and inhibitory signals. This may provide a novel molecular target for inducing immunotolerance but needs further study.
\end{abstract}

Keywords: NK92, GST-sHLA-G1 $\alpha$ chain, signal transduction, SHP-1, O-GlcNAc.

\section{INTRODUCTION}

Natural killer (NK) cells are large granular lymphocytes of the innate immune system that are especially abundant in the placenta and are also known as "uterine granular lymphocytes"[1]. According to the "missing self" hypothesis [2], NK cells will recognize and eliminate those cells who fail to express self major histocompatibility complex (MHC) class I molecules. But why the maternal NK cells can tolerate semiallogenenic fetal tissues on which do not express maternal MHC class I molecules? Human leukocyte antigen-G (HLA-G), a nonclassical MHC I molecule, which is predominantly expressed by fetal extravillous trophoblasts, may be the answer[3]. Unlike classical MHC I antigens, HLA-G exhibits a tissue-restricted distribution pattern and its mRNA is alternatively spliced to encode seven different isoforms, namely the four membranebound proteins, HLA-G1, -G2, -G3, -G4 and three soluble

\footnotetext{
*Co-correspondence: Mei Fu FENG, Tel/Fex: +86 1062571017 , E-mail: fengmf@panda.ioz.ac.cn,

and Rou Li ZHOU, Tel: +86 1082801476 , Fax: +86 1082358270 , E-mail: rlzhou@bjmu.edu.cn

Abbreviations: HLA, human leukocyte antigen (HLA); NK, natural killer cells (NK); O-GlcNAc, O-linked $\beta$-N-acetylglucosamine (O-GlcNAc); SHP-1, Src homology 2 domain-containing tyrosine phosphatase-1.
}

isoforms, HLA-G5 (also termed sHLA-G1), -G6, -G7[45]. HLA-G plays an important role not only during pregnancy, but also during allograft and xenograft[6]. In addition, it provides new strategies to cure tumors and inflammation because HLA-G is also expressed in some tumors and inflamed cells[7-8].

Soluble HLA-G1 $\alpha$ chain (sHLA-G1 $\alpha$ chain), encoded by a mRNA retaining intron 4 , is a protein that lacks the transmembrane and cytoplasmic domains[9]. In physiological condition, a large quantity of sHLA-G1 is secreted in the amniotic fluid and released into the maternal peripheral blood where they may exhibit systemic immunoinhibitory functions[10]. It was shown that the soluble HLA-G could trigger CD95/CD95 ligand-mediated apoptosis in activated $\mathrm{CD}^{+}$cells[11] and suppress the allo-proliferative response [12]. These might be the important mechanisms implicated in the immunotolerance of the fetal allograft.

To date, three NK inhibitory receptors that directly interact with HLA-G have been identified on NK cells: (1) KIR2DL4 (p49)[13, 14], which is specific for HLA-G and is composed of two extracellular Ig-like domains and a single ITIM in its cytoplasmic tail; (2) ILT-2 (or LIR-1) [15], which belongs to a new family of Ig-SF-receptors and has four extracellular Ig domains and four ITIMs in 
its intracytoplasmic tail; (3) ILT-4[16], which is selectively expressed in monocytes, macrophages and dendritic cells (DCs), can bind to both classical class I molecules and nonclassical class I molecules HLA-G. Little is known about how soluble HLA-G interacts with its receptors on NK cells, and how the balance between activating and inhibitory signals in NK cells is kept. To answer these questions, the sHLA-G1 $\alpha$ chain was expressed as a fusion protein with glutathione S-transferase (GST) and the mechanism of inhibitory effect of this protein was studied.

\section{MATERIALS AND METHODS}

\section{Cell culture}

Human erythroleukemia HLA-A, -B, -C, and -G-negative K562 cells were maintained in RPMI 1640 medium with $10 \%$ fetal calf serum (Hyclone). NK cell line NK92 cells (provided by Immune Medicine Inc, Canada) were cultured in $\alpha$-MEM supplemented with $12.5 \%$ fetal calf serum, $12.5 \%$ equine serum, $0.2 \mathrm{mM}$ inositol, $0.1 \mathrm{mM} \beta$-mercaptoethanol, $0.02 \mathrm{mM}$ folic acid and 100-200 U/ml recombinant IL-2 (BD Pharmingen) at $37^{\circ} \mathrm{C}$ in a $5 \% \mathrm{CO}_{2}$ humidified incubator.

\section{The expression and purification of GST-sHLA-G1 $\alpha$ chain}

The extracellular domain of $H L A-G 1$ was ligated into a pGEX$4 \mathrm{~T} 2$ vector (Pharmacia Biotech). The new construct was sequenced to confirm the correct open-reading frame. E. coli BL21 cells were then transformed with the construct pGEX-4T2-sHLA-G1 $\alpha$ chain and the control vector pGEX-4T2 respectively. Isopropyl-beta Dthiogalactopyranoside (IPTG, GIBCO) was used to induce high production of control protein GST and target protein GST-sHLAG1 $\alpha$ chain. GST and GST-sHLA-G1 $\alpha$ chain products were recovered by gradient dialysis and purified with glutathione-sepharose affinity column according to manual. The target protein was confirmed by immunoblotting. The purity of target protein was determined by SDS-PAGE. Endotoxin content in testing system was determined by using Tachypleus Amebocyte Lysate Kit (Zhanjiang A\&C Biological Limited Company). Endotoxin standards were obtained from National Institute for the Control of Pharmaceutical and Biological Products in China.

\section{Cytotoxicity assay}

The $4 \mathrm{~h}{ }^{51} \mathrm{Cr}$-release assay was performed to determine the cytolytic activities of NK92 cells. K562 cells were used as target cells (T). The fusion protein GST-HLA-Gl $\alpha$ chain or the GST control was preincubated for $30 \mathrm{~min}$ at $4^{\circ} \mathrm{C}$ with NK92 cells before adding $5 \times 10^{3}$ ${ }^{51} \mathrm{Cr}$-labeled $\mathrm{K} 562$ cells $\left(100 \mu \mathrm{Ci}\right.$ of ${ }^{51} \mathrm{Cr}$ sodium chromate $/ 10^{6}$ cells, Amersham, UK) at different effector/target (E/T) ratios of 5:1 and 2.5:1. After $4 \mathrm{~h}$ incubation at $37^{\circ} \mathrm{C}, 50 \mu 1$ of the supernatant was collected for liquid scintillation counting (Wallac 1410, Pharmacia). Spontaneous release was measured in target cells incubated with media alone and maximal release was measured by treatment of target cells with $1 \%$ Triton X-100. Percent specific lysis was determined by the following formula: $\%$ specific lysis $=($ experimental release spontaneous release $) /($ maximum release - spontaneous release $) \times 100 \%$. Results were presented as means $\pm \mathrm{SD}$ of triplicate samples.

\section{Detection of receptor ILT-2 on NK92 cells}

NK92 cells were washed with PBS and incubated with the monoclonal antibody against ILT-2 (anti-CD85, BD Pharmingen) at $4{ }^{\circ} \mathrm{C}$ for $2 \mathrm{~h}$. After thoroughly washing, they were incubated with FITCgoat-anti-mouse IgG (Southern Biotechnology Associates) at $4{ }^{\circ} \mathrm{C}$ for $2 \mathrm{~h}$ and washed again, and the expression of ILT-2 was detected by flow cytometric analysis (FACSCalibur, BD, USA). Data was analyzed with Cell Quest 3.0 from BD Corporation.

\section{Fixation of target cells[17]}

K562 cells were washed with PBS and incubated with $1 \%$ paraformaldehyde on ice for $30 \mathrm{~min}$. Cells were then washed again to remove paraformaldehyde.

\section{Immunoprecipitation and Western blotting}

The NK92 cells were incubated with fixed K562 cells with or without the presence of GST-sHLA-G1 $\alpha$ chain respectively for 5 , $15,30,60$ min respectively. The cells were lysed and the debris was cleared. The lysate was then incubated with anti-ILT-2 antibody overnight and subsequently with protein A-agarose for $4 \mathrm{~h}$ at $4^{\circ} \mathrm{C}$. After washing, the beads were boiled in SDS buffer and the supernant was collected. All samples were separated by SDS-PAGE followed by Western blotting. Antibodies used were: $87 \mathrm{G}$, (a anti-sHLA-G1 $\mathrm{mAb}$, gifted by Dr. D. Geraghty, Fred Hutchinson Cancer Research Center, USA), PY20, a specific antibody against the phosphorylated tyrosine (Phosphotyrosine Ab-1, Neomarkers, USA), anti-phosphoMEK (Cell Signaling Technology, Inc), anti-MEK (Cell Signaling Technology, Inc), anti-phospho-ERK (Cell Signaling Technology, Inc), anti-ERK (sc-94, Santa Cruz Biotechnology, Inc), CTD110.6 (anti-O-GlcNAc, Covance, USA) and PTP1C/SHP1 (p17320, BD Transduction Laboratories). Proteins were detected by NBT and BCIP response.

\section{RESULTS}

\section{Identification of the GST-sHLA-G1 $\alpha$ chain fusion protein}

The $s H L A-G 1 \alpha$ chain gene sequence was identical to the extracellular fragment of HLA-G1 $\alpha$ chain whose gene bank number is AF226990. BL21 cells transformed with the pGEX-4T2-sHLA-G1 $\alpha$ chain vector or the pGEX-4T2 control vector were induced with IPTG, and the bacterial protein products were examined. Coomassie blue-stained sodium dodecyl sulfate-polyacrylamide gel electrophoresis (SDS-PAGE) of induced or uninduced cell lysates showed that $59 \mathrm{kD}$ fusion protein was overproduced in the pGEX-4T2-sHLA-G1 $\alpha$ chain transformed BL21 cells (Fig 1A), while the pGEX-4T2 transformants produced only a $29 \mathrm{kD}$ GST protein. Western blot profiles contained a band corresponding to the $59 \mathrm{kD}$ protein, which was specifically recognized by $87 \mathrm{G} \mathrm{mAb}[12]$, a specific antibody against sHLA-G1 (Fig 1B), confirming that the fusion protein was derived from the $H L A-G 1 \alpha$ chain coding sequence. Soluble target protein was purified by glutathione-sepharose affinity column to $98 \%$ purity determined 


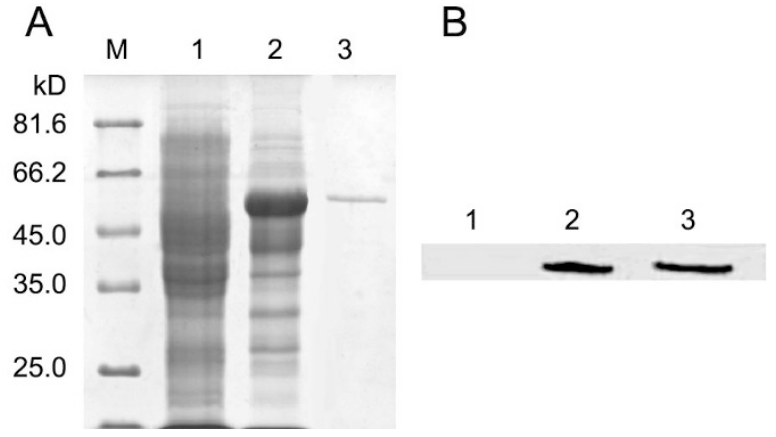

Fig 1. Detection of GST-sHLA-G1 $\alpha$ chain by SDS-PAGE and Western blotting. (A) Detection of GST-sHLA-G1 $\alpha$ chain by $12 \%$ SDSPAGE. (B) Detection of GST-sHLA-G1 $\alpha$ chain by Western blotting with antibody $87 \mathrm{G}$. Lane 1 , total cell proteins before induction. Lane 2 , total cell proteins after induction with IPTG. Lane 3, GST-sHLAG1 $\alpha$ chain eluted from gluthathione-sepharose column. M, standard protein.

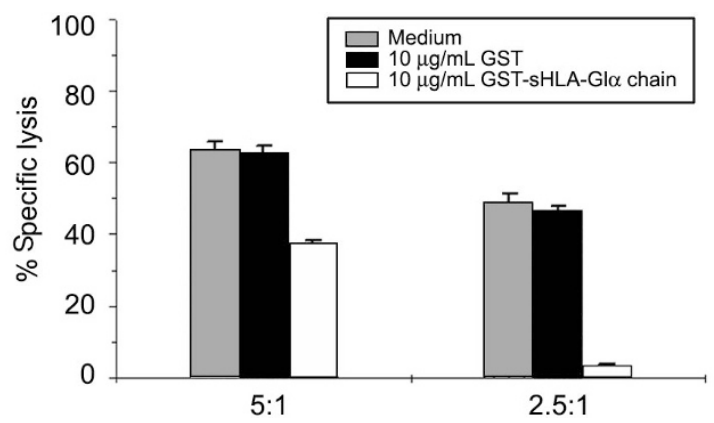

Fig 2. Effect of GST-sHLA-G1 $\alpha$ chain on the cytotoxic activity of NK92 cells. Purified GST-sHLA-G1 $\alpha$ chain and GST were used at indicated concentrations. Results were presented as the percentage lysis in a $4 \mathrm{~h}{ }^{51} \mathrm{Cr}$-release assay. At the E/T ratio of $5: 1,10 \mu \mathrm{g} / \mathrm{ml}$ GST-sHLA-G1 $\alpha$ chain could decrease NK92 cytotoxic activity compared to medium and GST (control) groups. At the $(\mathrm{E} / \mathrm{T})$ ratio of $2.5: 1$, $10 \mu \mathrm{g} / \mathrm{ml} \mathrm{GST-sHLA-G1} \alpha$ chain protein was able to inhibit NK92 cytotoxic activity.

by SDS-PAGE. Immunoblots showed that the purified protein was GST-sHLA-G1 $\alpha$ chain. The final endotoxin content was $2.2 \mathrm{EU} / \mathrm{mg}$ of protein.

\section{Inhibition of NK92 Cytotoxicity by GST-sHLA-G1 $\alpha$ chain}

The $4 \mathrm{~h}{ }^{51} \mathrm{Cr}$-release assay showed that $10 \mu \mathrm{g} / \mathrm{ml} \mathrm{GST}$ sHLA-G1 $\alpha$ chain protein was able to inhibit NK92 cytolytic activity at the E/T ratio of 2.5:1, while the control GST protein could not (Fig 2).

\section{Detection of the ILT-2 receptor by flow cytometric analysis}

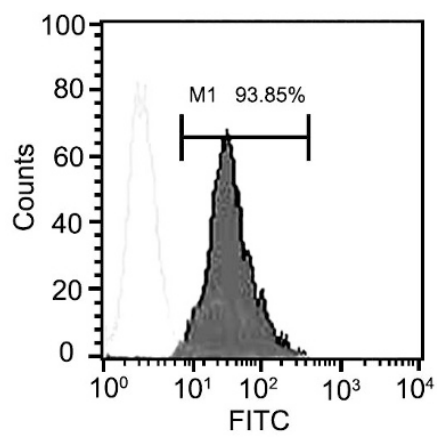

Fig 3. Flow cytometry analysis of ILT-2 receptor on NK92 cell surface. ILT-2 receptor expression was detected by flow cytometry analysis using anti-CD85 antibody and FITC-goat-anti-mouse IgG. Left: control, which was NK92 cells without incubation with antibody, M1: the expression ratio of ILT-2 on NK92 cells, which was $93.85 \%$.

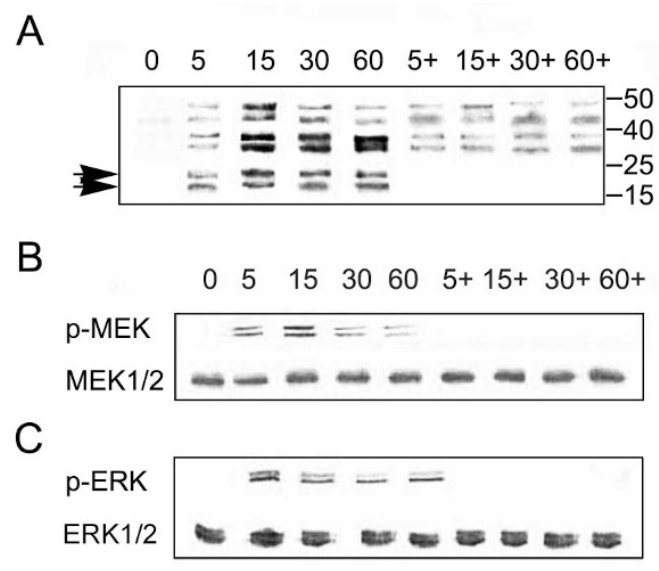

Fig 4. Inhibition of the activating signals in NK92 cells by GSTsHLA-G1 $\alpha$ chain. NK92 cells interacted with K562 for 0-60 min. + , the presence of GST-sHLA-G1 $\alpha$ chain in the incubation system. (A) Detection of tyrosine phosphorylation by Western blotting with PY20. Arrowheads represent some proteins whose phosphorylation was completely inhibited by GST-sHLA-G1 $\alpha$ chain. M, the protein markers. (B) Detection of phosphorylated MEK1/2 by Western blotting with anti-p-MEK (top). The blots were probed with anti-MEK1/2 to show equal loading of all lanes (bottom). (C)

Three HLA-G inhibitory receptors have been identified on NK cells: KIR2DL4, ILT-2, ILT-4. ILT-2 can be identified by anti-CD 85 antibody. The flow cytometric analysis result showed that ILT-2 was expressed on NK92 cell line (Fig 3).

\section{Effect of GST-sHLA-G1 $\alpha$ chain on the MEK-ERK pathway in NK92 cells}

The protein-tyrosine kinases (PTKs) have been proved to provide early and requisite signals for cytotoxic function $[18,19]$. Since the phosphorylation of tyrosine can represent PTK activity, we next examined the effect of 
GST-sHLA-G1 $\alpha$ chain on the tyrosine phosphorylation in NK92 cells induced by susceptible targets. Western blot results showed that tyrosine phosphorylation increased rapidly and reached its peak at $15 \mathrm{~min}$ after stimulation with K562 cells (Fig 4A).

Mostly the phosphorylation of tyrosine kinases triggers downstream events via Raf-MEK-ERK pathways. Downstream molecules, MEK and ERK were essential to regulate perforin and granzyme B mobilization and redistribution towards the contact zone between NK cells and target cells during NK cell cytotoxicity[20]. The MEK-ERK pathway was rapidly activated upon target engagement in human NK92 cells and played an important role in cytotoxicity of NK92 cells. We found that GST-sHLA-G1 $\alpha$ chain inhibited some protein tyrosine phosphorylation (Fig 4A, bands indicated by arrowheads) and subsequently blocked the activation of MEK-ERK (Fig 4B, 4C).

\section{Recruitment of SHP-1 by ILT-2 receptor}

ILT-2 has four immunoreceptor tyrosine-based inhibition motifs (ITIMs)[21], with consensus sequence (I/V) $\mathrm{xYxx}(\mathrm{L} / \mathrm{V})$ contributing to the recruitment of Src homology 2 domain-containing tyrosine phosphatases (SHP). GST-sHLA-G1 $\alpha$ chain could bind to the receptor ILT-2 and then ILT-2 recruited $\mathrm{SH} 2$ domain-containing protein tyrosine phosphatase 1 (SHP-1) (Fig 5). In contrast, SHP-1 recruitment was not observed in NK92 cells alone or NK92 cells which were incubated with K562 cells. The results showed that GST-sHLA-G1 $\alpha$ chain could bind to the receptor ILT-2 and the latter recruited SHP-1 which dephosphorylated some important PTKs and blocked the activation of downstream molecules such as MEK and ERK to inhibit the cytotoxicity of NK92 cells.

\section{Effect of GST-sHLA-G1 $\alpha$ chain on the O-GIcNAc modification in NK92 cells}

The dynamic glycosylation of serine or threonine residues on nuclear and cytosolic proteins by O-linked $\beta-\mathrm{N}-$ acetylglucosamine (O-GlcNAc) is adundant in all multicellular eukaryotes and O-GlcNAc modification has been reported to be a new regulatory modification important to signal transduction cascades[22]. To the best of our

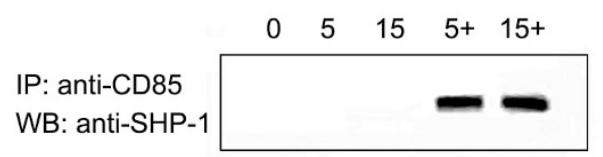

Fig 5. Recruition of SHP-1 by ILT-2 receptor. The cell lysates were immunoprecipitated with anti-human ILT-2 Ab (anti-CD85). Western blots were probed with anti-SHP-1 Ab. 0 represents sample from NK92 cells without stimulation. Numbers, the time after stimulation by K562 cells. +, NK92 cells incubated with GST-sHLA-G1 $\alpha$ chain.

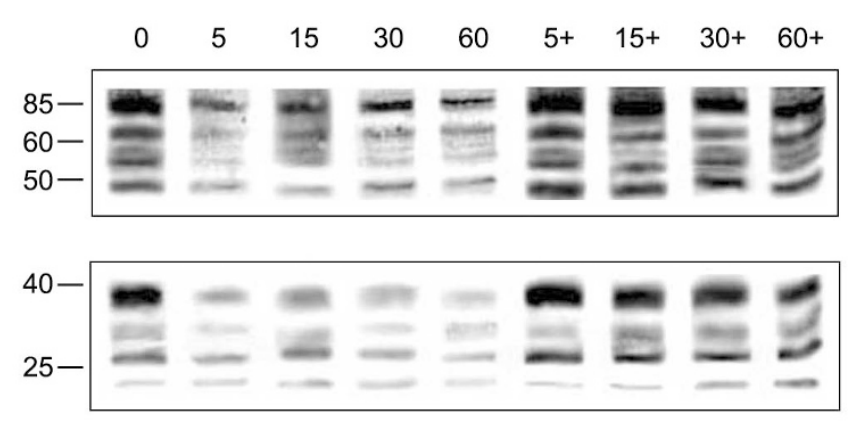

Fig 6. Detection of the O-GlcNAc modification in NK92 cells. Whole cell lysates were analyzed by Western blotting with CTD110.6. + represents the system with the presence of GST-sHLA-G1 $\alpha$ chain. Numbers represent the time after stimulation by K562 cells.

knowledge, our results showed that O-GlcNAc modification decreased during NK cell cytotoxicity and that GSTsHLA-G1 $\alpha$ chain could inhibit this decrease (Fig 6), indicating that $\mathrm{O}-\mathrm{GlcNAc}$ might get involved in the signal transduction of NK cells.

\section{DISCUSSION}

GST-sHLA-G1 $\alpha$ chain was produced as a fusion protein in $E$. coli and recognized by the anti-sHLA-G1 antibody, $87 \mathrm{G} \mathrm{mAb}$. According to the cytotoxic assays, this soluble GST-sHLA-G1 $\alpha$ chain protein was able to inhibit NK92 cytotoxic activity at the effector / target (NK92/K562) ratio of 2.5:1, while the control GST protein could not, which indicates that the inhibition of NK92 cytotoxic activity was exerted by GST-sHLA-G1 $\alpha$ chain, but not by GST fragment. The current study afforded a recombinant protein with biologic activity and lower endotoxin content. Furthermore, this GST-sHLA-G1 $\alpha$ chain fusion protein was not glycosylated or conjugated with $\alpha_{2}$ microglobulin. The results showed that in addition to membrane-bound HLA-G1 molecules, the soluble GST-sHLA-G1 $\alpha$ chain could also induce immunotolerance by inhibiting NK cell cytotoxicity.

In this study, ILT-2, a HLA-G receptor, was found to be expressed on NK92 cells by flow cytometry. In addition, NK92 cells also expressed an endogenous KIR2DL4 at low level[14]. This GST-sHLA-G1 $\alpha$ chain may also be useful for identifying new HLA-G receptors on NK cells. MHCrecognizing receptors have been reported to recruit SHP1 or SHP-2 to dephosphorylate[23]. Our results showed that GST-sHLA-G1 $\alpha$ chain bound to ILT-2 and then the latter recruited SHP-1 to dephosphorylate some important and early signal molecules such as PTKs so that the downstream MEK-ERK pathway was consequently blocked.

The balance of activating and inhibitory signal transduction of NK cells is a complicated multifactorial process. 
Recently O-GlcNAc modification has been reported to be involved in regulating signal transduction in eukaryotes[24]. Regulation of O-GlcNAc levels by O-GlcNAc transferase and GlcNAcase may be analogous to the regulation of phosphorylation and dephosphorylation by kinases and phosphatases. O-GlcNAc modification of certain proteins is known to fluctuate during $\mathrm{T}$ cell activation, insulin signaling, glucose metabolism, and cell cycle progression [24]. For examples, the elevation of O-GlcNAc levels was associated with insulin resistance in adipocytes[25] and the O-GlcNAc modification of tau, $\mathrm{AP}_{3}$ and so on was perturbed in Alzheimers disease patients[26]. In the present study, we reported that O-GlcNAc modification was involved in cytotoxic signal transduction in NK cells and that GST-sHLA-G1 $\alpha$ chain could inhibit the alteration of O-GlcNAc level in NK cells. Several groups have documented reciprocity between O-GlcNAc and O-phophorylation[24]. A given serine/threonine may exist in three states: glycosylated, phosphorylated, or unmodified. O-GlcNAc could have an effect by blocking phosphorylation and/or by an independent mechanism, such as the modulation of protein-protein interactions. Which is the real one in NK cells during cytotoxic activity is under further study.

In conclusion, the GST-sHLA-G1 $\alpha$ chain was produced and its function was studied. It could inhibit NK cell cytotoxicity by interacting with receptor ILT- 2 and consequently recruiting SHP-1, blocking some protein tyrosine phosphorylation, inhibiting the activation of MEK and ERK signaling pathway. The results also showed that the modification of O-GlcNAc was involved in NK activating and inhibitory signals. Therefore, further studies are needed not only on the signaling pathway initiated by receptors but also how the multiple signals are integrated for regulation of NK cell responses.

Received, April 4, 2003

Revised, Mar 21, 2004

Accepted, Mar 25, 2004

\section{REFERENCES}

1 King A, Loke YW. On the nature and function of human uterine granular lymphocytes. Immunol Today 1991; 12:432-5.

2 Ljunggren HG, Karre K. In search of the 'missing self': MHC molecules and NK cell recognition. Immunology Today 1990; 11:237-44.

3 Carosella ED, Rouas-Freiss N, Paul P, et al. HLA-G: a tolerance molecule from the major histocompatibility complex. Immunol Today 1999; 20:60-2.

4 Bainbridge D, Ellis S, Le P, et al. HLA-G remains a mystery. TRENDS Immunol 2001; 22(10):548-52.

5 Paul P, Cabestre FA, Ibrahim EC, et al. Identification of HLAG7 as a new splice variant of the HLA-G mRNA and expression of soluble HLA-G5, -G6, and -G7 transcripts in human transfected cells. Hum Immunol 2000; 61(11):1138-49.
6 Matsunami K, Miyagawa S, Nakai R, et al. Protection against natural killer-mediated swine endothelial cell lysis by HLA-G and HLA-E. Transplant Proc 2000; 32:939-40.

7 Paul P, Adria'n Cabestre'F, Le Gal F, et al. Heterogeneity of HLA-G Gene Transcription and Protein Expression in Malignant Melanoma Biopsies. Cancer Res 1999; 59:1954-60.

8 Onno M, Le FG, Pangault C, et al. Modulation of HLA-G antigens expression in myelomonocytic cells. Hum Immunol 2000; 61(11):1086-94 .

9 Fujii, T, Ishitani A, Geraghty DE. A soluble form of the HLA-G antigen is encoded by a messenger ribonucleic acid containing intron 4. J Immunol 1994; 153(12):5516-24.

10 McMaster M, Zhou Y, et al. HLA-G isoforms produced by placental cytotrophoblasts and found in amniotic fluid are due to unusual glycosylation. J Immunol 1998; 160(12):5922-8.

11 Fournel Y, Aguerre-Girr M, Huc X, et al. Cutting edge: soluble HLA-G1 triggers CD95/CD95 ligand-mediated apoptosis in activated CD8 ${ }^{+}$Cells by interacting with CD8. J Immunol 2000; 164:6100-4.

12 Lila N, Rouas-Freiss N, Dausset J, et al. Soluble HLA-G protein secreted by allo-specific $\mathrm{CD} 4^{+} \mathrm{T}$ cells suppresses the allo-proliferative response: a $\mathrm{CD} 4^{+} \mathrm{T}$ cells regulatory mechanism. Proc Natl Acad Sci USA 2001; 98(21):12150-5.

13 Cantoni C, Verdiani S, Falco M, et al. p49, a putative HLA class I-specific inhibitory NK receptor belonging to the immunoglobulin superfamily. Eur J Immunol 1998; 28(6):1980-90.

14 Rajagopalan S, Long EO. A human histocompatibility leukocyte antigen (HLA)-G specific receptor expressed on all natural killer cells. The Journal of Experimental Medicine 1999; 189(7):1093-9.

15 Navarro F, Llano M, Bellón T, et al. The ILT2(LIR1) and CD94/ NKG2A NK cell receptors respectively recognize HLA-G1 and HLA-E molexules co-expressed on target cells. Eur J Immunol 1999; 29:277-83.

16 Colonna M, Samaridis J, Cella M, et al. Human myelomonocytic cells express an inhibitory receptor for classical and nonclassical MHC class I molecules. The J Immunol 1998; 160:3096-100.

17 Wei S, Gilvary DL, Corliss BC, et al. Direct tumor lysis by NK Cells uses a Ras-independent mitogen-activated protein kinase signal pathway. J Immunol 2000; 165:3811-9.

18 Azzoni L, Kamoun M, Salcedo TW, et al. Stimulation of Fc $\gamma R$ IIIA results in phospholipase C- $\gamma 1$ tyrosine phosphorylation and p56lck activation. J Exp Med 1992; 176:1751-5.

19 Kanakaraj P, Duckworth B, Azzoni L, et al. Phosphatidylinositol3 kinase activation induced upon Fc $\gamma$ RIIIA interaction. J Exp Med 1994; 179:551-8.

20 Wei S, Gamero AM, Liu JH, et al. Control of lytic function by mitogen-activated protein kinase/extracellular regulatory kinase2 (ERK2) in a human natural killer cell line: idenification of perforin and granzyme B mobilization by functional ERK2. J Exp Med 1998; 187:1753-65.

21 Vivier E, Daëron M. Immunoreceptor tyrosine-based inhibition motifs. Immunol Today 1997; 18(6):286-91.

22 Well L, Gao Y, Mahoney JA, et al. Dynamic o-glycosylation of nuclear and cytosolic proteins. J Biol Chem 2002; 277(3):1755-61.

23 Gupta N, Scharenberg AM, Burshtyn DN, et al. Negative signaling pathways of the killer cell inhibitory receptor and $\mathrm{Fc}$ gamma Rllb1 require distinct phosphateses. J Exp Med 1997; 186:473-8. 24 Well L, Vosseller K, Hart GW, et al. Glycosylation of nucleocy- 
toplasmic proteins: signal transduction and O-GlacNAc. Science 2001; 291(5512):2376-8.

25 Vosseller K, Wells L, Lance DM, Hart GW. Elevated nucleoplasmic glycosylation by O-GlcNAc results in insulin resistance associated with defects in Akt activation in 3T3-L1 adipocytes.
Proc Natl Acad Sci USA 2002; 99(8):5313-8.

26 Griffith LS, Schmitz B. O-linked N-acetylglucosamine is upregulated in Alzheimer brains. Biochem Biophys Res Commun 1995; 213:424-31. 